\title{
COHOMOLOGY AND DEFORMATIONS OF ALGEBRAIC STRUCTURES
}

\author{
BY ALBERT NIJENHUIS AND R. W. RICHARDSON, JR. ${ }^{1}$ \\ Communicated by Raoul Bott, December 19, 1963
}

Gerstenhaber has recently initiated a theory of deformations of associative algebras [4]. The methods and results of Gerstenhaber's work are strikingly similar to those in the theory of deformations of complex analytic structures on compact manifolds. In this note we shall indicate how some of Gerstenhaber's ideas can be reformulated within a framework designed to exploit this similarity, that of graded Lie algebras.

In the work of Kodaira-Nirenberg-Spencer [7] and Kuranishi [8] on the existence of deformations of complex analytic structures, a basic role is played by a certain equation $[7$, p. 452 , equation (3)'] among the 1-dimensional elements of a graded Lie algebra; this equation expresses the integrability conditions for almost complex structures. Our basic observation is that a wide class of algebraic structures on a vector space can be defined by essentially the same equation in an appropriate graded Lie algebra. Among the structures thus obtained are Lie algebras, associative algebras, commutative and associative algebras, extensions of algebras (of any of the above types), and representations of algebras. Our main result, Theorems B and C, is a precise algebraic analogue of a theorem of Kuranishi [8] on deformations of complex analytic structures. Roughly speaking, it states that the set of all structures near a given one can be described in terms of certain cohomology groups, which are defined naturally by means of the graded Lie algebra. In some of the cases mentioned above, these cohomology groups are standard in homological algebra, in others they seem to be new. We also obtain an analogue, Theorem A, of the rigidity theorem of Frölicher and Nijenhuis [2] for deformations of complex analytic structures.

This note contains only an outline of results. A detailed exposition will appear elsewhere.

1. Graded Lie algebras and cohomology. Let $K$ be a field of characteristic $\neq 2$. Let $E=\sum_{n \geqq 0} E^{n}$ be a graded vector space over $K$. Let

1 The authors acknowledge partial support received from an Office of Naval Research contract at the University of Washington. The first named author is a Fulbright Fellow at the University of Amsterdam and also received partial support from NSF GP 1905 at the University of Pennsylvania. The second named author is an NSF Postdoctoral Fellow. 
$(x, y) \rightarrow[x, y]$ be a bilinear map of $E \times E$ into $E$. Then $(E,[]$,$) is a$ graded Lie algebra if the following conditions are satisfied:

(1) if $x \in E^{m}$ and $y \in E^{n}$, then $[x, y] \in E^{m+n}$,

(2) if $x \in E^{m}$ and $y \in E^{n}$, then $[x, y]=-(-1)^{m n}[y, x]$, and

(3) if $x \in E^{m}, y \in E^{n}$, and $z \in E^{p}$, then

$$
(-1)^{m p}[[x, y], z]+(-1)^{n m}[[y, z], x]+(-1)^{p n}[[z, x], y]=0 \text {. }
$$

The equation given in condition (3) is called the Jacobi identity.

Let $E$ be a graded Lie algebra and let $M=\left\{x \in E^{1} \mid[x, x]=0\right\}$. For each $x \in E$, let $\delta_{x}$ denote the linear map $y \rightarrow[x, y]$ of $E$ into $E$. If $x \in M$, it follows from the Jacobi identity that $\delta_{x} \circ \delta_{x}=0$. Thus the graded vector space, $E$, with the differentiation operator $\delta_{x}$, is a cochain complex. In the standard manner we define $Z^{n}(x)$ $=E^{n} \cap$ kernel $\left(\delta_{x}\right), B^{n}(x)=\delta_{x}\left(E^{n-1}\right)$, and $H^{n}(x)=Z^{n}(x) / B^{n}(x)$. Let $H(x)$ denote the direct sum of the vector spaces $\left(H^{n}(x)\right)_{n \geqq 0}$. It follows from the Jacobi identity that $\delta_{x}$ is a derivation of degree 1 of $E$. Thus $H(x)$ inherits from $E$ the structure of a graded Lie algebra.

Let $E=\sum_{n \geq 0} E^{n}$ be a graded Lie algebra and let $D^{0}(E)$ denote the vector space of all derivations of degree 0 of $E$. Then $D^{0}(E)$ is a Lie algebra with the commutator defined in the usual manner. Further $E^{0}$ is a Lie algebra and the map $x \rightarrow \delta_{x}$ is a homomorphism of the Lie algebra $E^{0}$ into the Lie algebra $D^{0}(E)$.

Let $E$ be a finite-dimensional graded Lie algebra. Suppose that we are given: (1) an irreducible algebraic linear group $G$ with Lie algebra (isomorphic to) $E^{0}$; and (2) an everywhere defined rational representation $\rho$ of $G$ by automorphisms (of degree 0 ) of the graded Lie algebra $E$, whose differential is the map $x \rightarrow \delta_{x}$. If these conditions are satisfied, we say that $E(=(E, G, \rho))$ is an algebraic graded Lie algebra.

Let $E$ be an algebraic graded Lie algebra and let $M=\left\{x \in E^{1} \mid[x, x]\right.$ $=0\}$. Then it follows easily that $M$ is stable under the action of $G$, and we can form the space of orbits $M / G$. The purpose of this paper is to show how one can obtain information on the local structure of $M / G$ in terms of the cohomology groups defined by elements of $M$.

The reason for considering graded Lie algebras in this generality is that a wide class of algebraic structures can be described in terms of the formalism above. Let $V$ be a finite-dimensional vector space over $K$. Then there exists an algebraic graded Lie algebra $A(V)$ (to be described in the following section) such that the points of $M$ (defined as above) are precisely the Lie algebra multiplications on $V$. Furthermore two points of $M$ lie on the same orbit under $G$ if and only if the corresponding Lie algebras are isomorphic. If $x$ is a point of $M$ and if $L$ is the corresponding Lie algebra, then the cohomology group 
$H^{n}(x)$ is isomorphic with the Lie algebra cohomology group $H^{n+1}(L, L)$ as defined by Chevalley and Eilenberg [1]. Similar remarks hold for the case of associative (resp. associative and commutative) multiplications on $V$. In this case the cohomology groups are those defined by Hochschild [6] (resp. Harrison [5]). The existence of a graded Lie algebra structure on $H(L, L)\left(=\sum H^{n}(L, L)\right)$ is due to Gerstenhaber [3].

If $A$ and $B$ are finite-dimensional algebras (either Lie or associative or associative and commutative) over $K$, then there exists an algebraic graded Lie algebra such that the set $M$ (defined by essentially the same equation as above) is the set of all extensions of $A$ by $B$, and such that two points of $M$ lie on the same orbit under $G$ if and only if the corresponding extensions are equivalent. Similarly there exists an algebraic graded Lie algebra which describes the set of all representations of a given algebra on a given vector space. The cohomology groups which arise in these last two cases seem to be new.

2. The graded Lie algebra of alternating multilinear maps. As an example we describe the graded Lie algebra which is associated with Lie algebra multiplications on a given vector space. Let $V$ be a vector space over $K$. For each integer $n \geqq 0$, let $A^{n}(V)$ denote the vector space of all alternating multilinear maps of $V^{n+1}$, the $(n+1)$-fold Cartesian product of $V$ with itself, into $V$. Let $A(V)$ denote the direct sum of the family of vector spaces $\left(A^{n}(V)\right)_{n \geq 0 .}$. If $f \in A^{p}(V)$ and $h \in A^{q}(V)$, we define an element $f \pi h$ of $A^{p+q}(V)$ by

$$
\begin{array}{r}
f \pi h\left(u_{0}, \cdots, u_{p+q}\right) \\
\quad=\sum_{\eta} \operatorname{sgn}(\eta) f\left(h\left(u_{\eta(0)}, \cdots, u_{\eta(p)}\right), u_{\eta(p+1)}, \cdots, u_{\eta(p+q)}\right)
\end{array}
$$

where the sum is taken over all permutations $\eta$ of $\{0, \cdots, p+q\}$ such that $\eta(0)<\cdots<\eta(p)$ and $\eta(p+1)<\cdots<\eta(p+q)$. We define

$$
[f, h]=f \pi h-(-1)^{p q} h \pi f .
$$

Lemma. $A(V)$, with the multiplication given by $[$,$] , is a graded Lie$ algebra.

The proof is straightforward, but tedious.

Let $f \in A^{1}(V) ; f$ is thus an alternating bilinear map of $V \times V$ into $V$. We have

$$
[f, f](u, v, w)=2(f(f(u, v), w)+f(f(v, w), u)+f(f(w, u), v)) .
$$

Hence, remembering that characteristic $K \neq 2$, it follows that $(V, f)$ 
is a Lie algebra if and only if $[f, f]=0$. Let $M=\left\{f \in A^{1}(V) \mid[f, f]=0\right\}$. The points of $M$ are precisely the Lie algebra multiplications on $V$. Let $f \in M$ and let $L$ denote the Lie algebra $(V, f)$. Then a computation shows that the cohomology groups $H^{n}(f)$ (defined as in $\S 1$ ) are identical with the cohomology groups $H^{n+1}(L, L)$ as defined by Chevalley-Eilenberg. For each $g \in G L(V)$, let $T_{0}$ denote the induced linear map of $A(V)$ into $A(V)$. Then it is easily checked that the map $g \rightarrow T_{\theta}$ is a homomorphism of $G L(V)$ into the group $\operatorname{Aut}^{0}(A(V))$ of all degree preserving automorphisms of the graded Lie algebra $A(V)$. Furthermore two Lie algebra structures on $V$ are isomorphic if and only if the corresponding points of $M$ lie on the same orbit under $G L(V)$. If $V$ is finite dimensional, then $A(V)$ is finite dimensional, $G L(V)$ is an algebraic linear group with Lie algebra $A^{\circ}(V)$, and $g \rightarrow T_{0}$ is an everywhere defined rational representation of $G L(V)$. It is easily checked that in this case $A(V)$ is an algebraic graded Lie algebra.

3. The rigidity theorem. For the rest of the paper $E$ will denote an algebraic graded Lie algebra with associated algebraic linear group $G$ and $M$ will denote the algebraic set $\left\{x \in E^{1} \mid[x, x]=0\right\}$.

Definition. An element $a \in M$ is rigid if the orbit $G(a)$ is a Zariski open subset of $M$.

Theorem A. Let $K$ be algebraically closed. If $a \in M$ and if $H^{1}(a)=0$, then $a$ is rigid.

A related theorem was proved for the case of associative algebras by Gerstenhaber [4].

The proof of Theorem A involves standard theorems in the theory of algebraic linear groups, but is otherwise quite elementary. This theorem implies, for example, that every semi-simple Lie algebra over the field of complex numbers is rigid. Furthermore the Lie algebra of all endomorphisms of a finite-dimensional complex vector space is rigid.

We note explicitly that the converse of Theorem A does not hold. However we do not know of any counterexamples to the converse for the graded Lie algebra $A(V)$.

4. An algebraic analogue of Kuranishi's theorem. (Added in proof.) In this section $E$ will denote an algebraic graded Lie algebra over either the real or complex field and we shall use transcendental methods (although of a very elementary nature). We shall consider $E$ as a topological space, supplied with the usual topology of a finitedimensional real or complex vector space. 
Theorem B. Let $E$ be an algebraic graded Lie algebra over either the real or complex field, let $M=\left\{x \in E^{1} \mid[x, x]=0\right\}$, and let $a \in M$. For each integer $j \geqq 0$, let $E_{j}^{1}=B^{i}(a)$, let $E_{0}^{j}$ be a supplementary subspace of $B^{i}(a)$ in $Z^{j}\left(=Z^{j}(a)\right)$, and let $E_{2}^{j}$ be a supplementary subspace of $Z^{i}$ in $E^{j}$. Let $\pi_{0}$ (resp. $\pi_{1}$ ) denote the projection of $E^{2}$ on $E_{0}^{2}$ (resp. $E_{1}^{2}$ ) with kernel $\left(E_{1}^{2}+E_{2}^{2}\right)$ (resp. $\left.\left(E_{0}^{2}+E_{2}^{2}\right)\right)$. Then there exist $N\left(a, Z^{1}\right)$ (resp. $\left.N\left(a, E^{1}\right)\right)$, a neighborhood of $a$ in $Z^{1}$ (resp. $\left.E^{1}\right)$, and analytic mappings $\phi: N\left(a, Z^{1}\right) \rightarrow E_{2}^{1}$, and $\psi: N\left(a, Z^{1}\right) \rightarrow E_{0}^{2}$, such that the following conditions hold:

(i) $\pi_{1}([x+\phi(x), x+\phi(x)])=0$,

(ii) $\psi(x)=\pi_{0}([x+\phi(x), x+\phi(x)])$,

(iii) $M \cap N\left(a, E^{1}\right)=\left\{x+\phi(x) \mid x \in N\left(a, Z^{1}\right)\right.$ and $\left.\psi(x)=0\right\}$.

We note that $E_{0}^{2}$ is naturally isomorphic to $H^{2}(a)$. Thus $\psi$ can be interpreted as a map from $N\left(a, Z^{1}\right)$ into $H^{2}(a)$. Theorem $\mathrm{B}$ says that $M$, in a neighborhood of $a$, can be parametrized by the subset $\psi^{-1}(0)$ of $Z^{1}(a)$. In particular if $H^{2}(a)=0$, then $M$ is locally parametrized by $Z^{1}(a)$.

The idea of the proof is as follows: Let $Q$ denote the map of $E^{1}$ into $E_{0}^{2}$ given by $x \rightarrow \pi_{1}([x, x])$. Write $E^{1}$ as $Z^{1} \times E_{2}^{1}$. Then in a neighborhood of $(a, 0)$ the "implicit equation" $Q(x, y)=0$ has a unique "solution" $y=\phi(x)$. The existence of $\phi$ follows from the implicit function theorem. The proof of (iii) uses the Jacobi identity and requires another application of the implicit function theorem.

Theorem C. Let $\beta=M \cap N\left(a, E^{1}\right)$. Let

$$
\varkappa=\left\{x+\phi(x) \mid x \in\left(N\left(a, Z^{1}\right) \cap\left(a+E_{0}^{1}\right)\right) \text { and } \psi(x)=0\right\} .
$$

Then there exists $N\left(0, E_{2}^{0}\right)$, a neighborhood of 0 in $E_{2}^{0}$, and a representation of $B$ as a product $\Re \times N\left(0, E_{2}^{0}\right)$, such that each set $\{k\} \times N\left(0, E_{2}^{0}\right)$ lies on an orbit of $G$.

Let $\alpha$ denote the map $x \rightarrow \zeta(a+x)$ of $E_{0}^{1}$ into $E_{0}^{2}$. Since $E_{0}^{1}$ (resp. $E_{0}^{2}$ ) is isomorphic to $H^{1}(a)$ (resp. $H^{2}(a)$ ), $\alpha$ can be interpreted as a map of $H^{1}(a)$ into $H^{2}(a)$. We see that $\mathcal{K}$ is naturally parametrized by the set $\alpha^{-1}(0)$. Furthermore $M$ can be represented, in a neighborhood of $a$, as a product of $\mathcal{K}$ and $N\left(0, E_{2}^{0}\right)$, where the second factor represents the action of $G$ on $M$. The properties of the set $\mathcal{K}$ are somewhat similar to those of a slice at $a$, as defined in the theory of (compact) transformation groups (see [9] for definitions).

The proof of Theorem $\mathrm{C}$ uses the inverse function theorem and properties of the exponential map of $E^{0}$ into $G$.

The set $\nVdash$ is a precise analogue, for the situations we have been 
considering, of the family of complex structures defined by Kuranishi in $[8]$.

\section{REFERENCES}

1. C. Chevalley and S. Eilenberg, Cohomology theory of Lie groups and Lie algebras, Trans. Amer. Math. Soc. 63 (1948), 85-124.

2. A. Frölicher and A. Nijenhuis, $A$ theorem on stability of complex structures, Proc. Nat. Acad. Sci. U.S.A. 43 (1957), 239-241.

3. M. Gerstenhaber, The cohomology structure of an associative ring, Ann. of Math. (2) 78 (1963), 267-289.

4. - On the deformation of rings and algebras, Ann. of Math. (2) 79 (1964), 59-104.

5. D. Harrison, Commutative algebras and cohomology, Trans. Amer. Math. Soc. 104 (1962), 191-204.

6. G. Hochschild, On the cohomology groups of an associative algebra, Ann. of Math. (2) 46 (1945), 58-67.

7. K. Kodaira, L. Nirenberg and D. Spencer, On the existence of deformation of complex analytic structures, Ann. of Math. (2) 68 (1958), 450-459.

8. M. Kuranishi, On the locally complete families of complex analytic structures, Ann. of Math. (2) 75 (1962), 536-577.

9. D. Montgomery and C. T. Yang, The existence of a slice, Ann. of Math. (2) 65 (1957), 108-116.

UNIVERSITY OF WASHINGTON 